\title{
Microgravity Geyser and Flow Field Prediction
}

\author{
J. I. Hochstein ${ }^{1}$, and J.G. Marchetta ${ }^{2}$ \\ University of Memphis, Memphis, TN, 38152 \\ R.J. Thornton ${ }^{3}$ \\ NASA Marshall Space Flight Center, Huntsville, AL, 35812
}

\begin{abstract}
Modeling and prediction of flow fields and geyser formation in microgravity cryogenic propellant tanks was investigated. A computational simulation was used to reproduce the test matrix of experimental results performed by other investigators, as well as to model the flows in a larger tank. An underprediction of geyser height by the model led to a sensitivity study to determine if variations in surface tension coefficient, contact angle, or jet pipe turbulence significantly influence the simulations. It was determined that computational geyser height is not sensitive to slight variations in any of these items. An existing empirical correlation based on dimensionless parameters was re-examined in an effort to improve the accuracy of geyser prediction. This resulted in the proposal for a re-formulation of two dimensionless parameters used in the correlation; the non-dimensional geyser height and the Bond number. It was concluded that the new non-dimensional geyser height shows little promise. Although further data will be required to make a definite judgement, the reformulation of the Bond number provided correlations that are more accurate and appear to be more general than the previously established correlation.
\end{abstract}

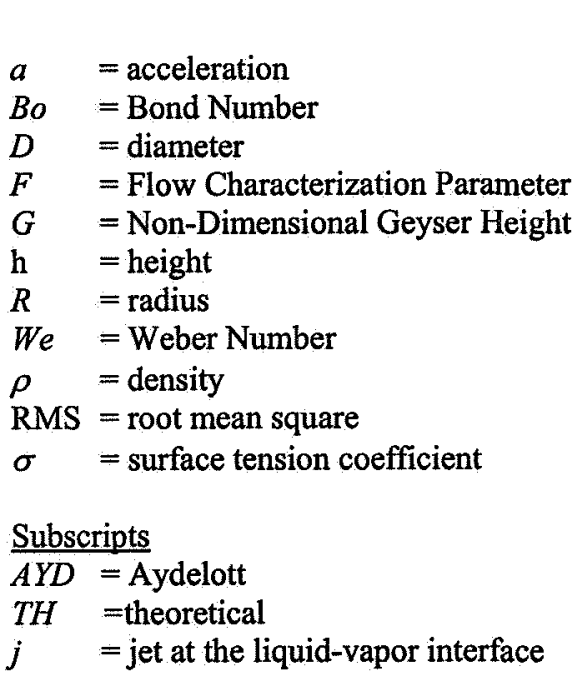

\section{Nomenclature}

\section{Introduction}

ONG duration spacecraft missions will require that rocket propellants be stored and maintained for future use. In the case of cryogenic liquid propellants, tank self-pressurization will be a significant problem. Despite insulation,

\footnotetext{
${ }^{1}$ Professor, Mechanical Engineering, 312 Engineering Sciences Bldg, AIAA Associate Fellow.

${ }^{2}$ Assistant Professor, Mechanical Engineering, 322D Engineering Sciences Bldg, AIAA Member.

${ }^{3}$ Mechanical Engineer, NASA Marshall Space Flight Center
} 
incident solar radiation heats the cryogenic fluid causing liquid to vaporize and raise the pressure inside the tank. If this self-pressurization were allowed to continue unchecked, the tank would rupture. A stronger tank could help, but this would require an undesirable increase in vehicle mass. Another solution to the tank pressurization problem is to vent the tank. Tank venting is undesirable because it wastes valuable propellant. In addition, due to the lack of gravity to positively orient the propellant in a predictable manner, it would be impossible to locate a vent where it could be certain that only vapor would be vented. In fact, even in partially filled tanks, it is possible that the entire tank surface could be wetted due to the influence of surface tension in a microgravity environment.

One alternative solution to the tank self-pressurization problem is a Therimodynamic Vent System, or TVS ${ }^{1,2}$. The TVS would extract a small portion of the bulk liquid from the tank and pass it through a Joule-Thomson valve resulting in a reduction in temperature as well as pressure. Once cooled, the fluid would be routed through a heat exchanger, which is used to cool a separate flow of propellant extracted from the bulk liquid. If the fluid leaving the Joule-Thomson valve is a two-phase mixture, it will continue changing phase in the heat exchanger until it is completely vaporized and will eventually be sacrificially vented overboard. The other stream of cooled liquid could then be pumped from the heat exchanger back into the tank and injected through an axial jet pipe located at the fore end of the tank. The jet flow provides several benefits including mixing of the bulk liquid, which helps to reduce temperature gradients and in turn helps prevent evaporation of the propellant ${ }^{3}$. Introduction of the cooled liquid also reduces the temperature of the bulk fluid. In addition, if the jet has a moderate amount of momentum it can cause the formation of a geyser at the liquid/vapor interface. The increased surface area of the free surface due to the formation of a geyser would help promote condensation, thus reducing the pressure even further. At higher levels of jet momentum, the geyser will strike the opposite end of the tank and either form a separate pool or "roll" down the tank walls re-mixing with the bulk fluid. As the fluid comes around the tank walls, there will also be a cooling effect on the wall. However, the addition of excessive kinetic energy to the bulk fluid would eventually result in undesirable heat generation through viscous dissipation.

A limited number of experiments have been conducted to investigate jet-induced mixing in reduced gravity. Aydelott ${ }^{1,2}$ performed the first small-scale drop-tower experiments using axial jets injected into four different tanks partially filled with ethanol. The four tanks were a spherical tank, a cylindrical tank with hemispherical heads, and two small-scale Centaur liquid hydrogen tanks. He identified the four distinct jet flow patterns presented in Fig. 1. Regime I occurs when the momentum is dissipated in the bulk liquid and the surface is not disturbed. Regime II 


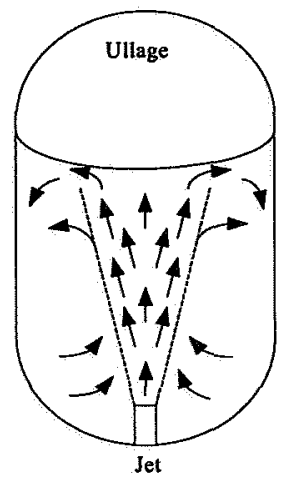

a) Pattern $I$.

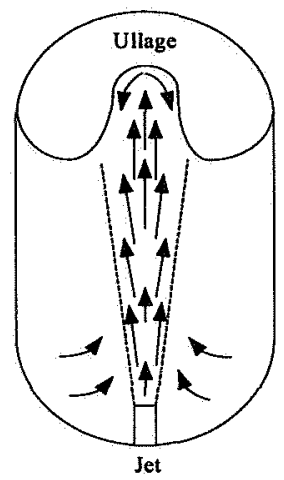

b) Pattern II.

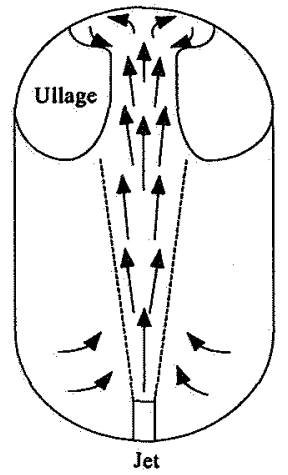

c) Pattern III.

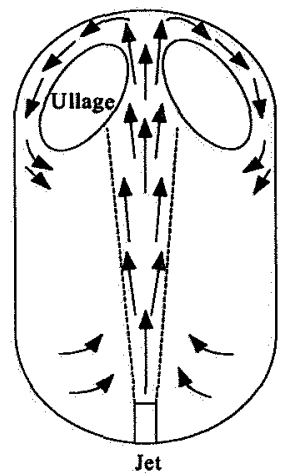

d) Pattern IV.

Fig. 1 Geyser Flow Patterns

occurs when the geyser forms at the surface, but does not reach the opposite end of the tank. Regime III occurs when the fluid reaches the opposite end of the tank and pools at that end. Regime IV occurs when the fluid reaches the opposite end of the tank and flows back along the walls of the tank, returning to the bulk fluid. Aydelott investigated mixing rates of the injected fluid and presents predicted flow patterns for partially and fully turbulent axial jets based on empirical correlations of the experiment data.

The ability to predict the flow pattern for any given combination of tank geometry, tank fill level, gravitational acceleration, and jet momentum is crucial for optimization and implementation of a TVS. A study of geysers and their formation is therefore a necessary task in the development process of the TVS concept. Thus, the focus of the present investigation is to enhance a computational model to simulate jet-induced mixing in reduced gravity and to validate the simulation results against the available Aydelott experiment data.

\section{Mathematical Formulation}

At the temperatures and pressures associated with propellant storage tanks, liquid propellants are well characterized as incompressible, constant property, Newtonian fluids. The two-dimensional Navier-Stokes equations, which describe the unsteady, turbulent flow of such a fluid expressed in cylindrical coordinates can be expressed in cylindrical coordinates as follows:

Continuity:

$$
\frac{1}{r} \frac{\partial\left(r u_{r}\right)}{\partial r}+\frac{\partial u_{z}}{\partial z}=0
$$


Momentum:

$$
\begin{aligned}
& \frac{\partial u_{r}}{\partial t}+u_{r} \frac{\partial u_{r}}{\partial r}+u_{z} \frac{\partial u_{r}}{\partial z}=-\frac{1}{\rho} \frac{\partial p}{\partial r}+g_{r}+v_{e f f}\left[\frac{\partial}{\partial r}\left(\frac{1}{r} \frac{\partial\left(r u_{r}\right)}{\partial r}\right)+\frac{\partial^{2} u_{r}}{\partial z^{2}}\right] \\
& \frac{\partial u_{z}}{\partial t}+u_{r} \frac{\partial u_{z}}{\partial r}+u_{z} \frac{\partial u_{z}}{\partial z}=-\frac{1}{\rho} \frac{\partial p}{\partial z}+g_{z}+v_{e f f}\left[\frac{1}{r} \frac{\partial}{\partial r}\left(r \frac{\partial u_{z}}{\partial r}\right)+\frac{\partial^{2} u_{z}}{\partial z^{2}}\right]
\end{aligned}
$$

The Jones Launder $k-\varepsilon$ model $^{4}$ with the Pope ${ }^{5}$ round jet correction term is used to model the turbulent jet and is presented in Eq. 3 This turbulence model utilizes the Boussinesq concept of a turbulent eddy viscosity, $v_{t}$, to model Reynolds stress, a term introduced into the momentum equations by the Reynolds-averaging process. The sum of the eddy viscosity and the molecular viscosity, $v$, is the effective viscosity.

Turbulent kinetic energy

$\frac{D k}{D t}=\frac{1}{r} \frac{\partial}{\partial r}\left(r\left(\frac{v_{f}}{\sigma_{k}}\right) \frac{\partial k}{\partial r}\right)+\frac{\partial}{\partial z}\left(\frac{v_{t}}{\sigma_{k}} \frac{\partial k}{\partial z}\right)+v_{t}\left\{2\left[\left(\frac{\partial u_{r}}{\partial r}\right)^{2}+\left(\frac{\partial u_{z}}{\partial z}\right)^{2}+\left(\frac{u_{r}}{r}\right)^{2}\right]+\left[\frac{\partial u_{r}}{\partial z}+\frac{\partial u_{z}}{\partial r}\right]^{2}\right\}-\varepsilon$

Dissipation Rate

$$
\begin{array}{r}
\frac{D \varepsilon}{D t}=\frac{1}{r} \frac{\partial}{\partial r}\left(r\left(\frac{v_{t}}{\sigma_{\varepsilon}}\right) \frac{\partial \varepsilon}{\partial r}\right)+\frac{\partial}{\partial z}\left(\frac{\nu_{t}}{\sigma_{\varepsilon}} \frac{\partial \varepsilon}{\partial z}\right)+C_{\varepsilon 1} v_{t} \frac{\varepsilon}{k}\left\{2\left[\left(\frac{\partial u_{r}}{\partial r}\right)^{2}+\left(\frac{\partial u_{z}}{\partial z}\right)^{2}+\left(\frac{u_{r}}{r}\right)^{2}\right]+\left[\frac{\partial u_{r}}{\partial z}+\frac{\partial u_{z}}{\partial r}\right]^{2}\right\}-C_{\varepsilon 2} \frac{\varepsilon^{2}}{k} \\
+\frac{1}{4} C_{\varepsilon 3} \frac{k^{2}}{\varepsilon}\left(\frac{u_{r}}{r}\right)\left(\frac{\partial u_{z}}{\partial r}-\frac{\partial u_{r}}{\partial z}\right)^{2}
\end{array}
$$

Kinematic Eddy Viscosity

$v_{t}=C_{\mu} \frac{k^{2}}{\varepsilon}$

Auxillary Relations and Coefficients

$C_{\varepsilon 1}=1.6 \quad C_{\varepsilon 2}=1.92 \quad C_{\varepsilon 3}=0.79 \quad C_{\mu}=0.09 \quad \sigma_{\mathrm{k}}=1.0 \quad \sigma_{\varepsilon}=1.3$

\section{Microgravity Jet-Induced Geyser Simulation}

\section{A. Computational Model}

The complexity and expense of microgravity experimentation severely limits the amount of data that can be, and has been, collected on microgravity geyser formation. Therefore, an attractive alternative is the use of CFD, computational fluid dynamics, to model the geyser flows. The use of a CFD code can significantly reduce the cost of investigating the microgravity geyser phenomena, as well as allow for easy manipulation of parameters such as tank size, fluid properties, jet momentum levels, and gravitational acceleration levels.

The ECLIPSE code, a variant of RIPPLE ${ }^{6}$, was used for the computational studies presented herein. ECLIPSE was chosen for its previously demonstrated ability to model transient, two-dimensional, laminar, incompressible 
fluid flows with free surfaces of general topology and to specifically model geyser flows in reduced gravity ${ }^{7-9}$ : A flow field is discretized into finite volumes to form a regular non-uniform mesh. ECLIPSE models free surfaces with volume of fluid (VOF) data on the mesh, and a continuum surface force (CSF) model is used to model surface tension. Staggered grid differential equation approximations result in a system of algebraic equations that are solved by a two step projection method employing an incomplete Cholesky conjugate gradient (ICCG) solution technique for the pressure Poisson equation (PPE). One of the features added to RIPPLE to produce ECLIPSE is the implementation of the two-equation Jones-Launder-Pope $k-\varepsilon$ turbulence model.

To simulate jet flows, several types of boundary conditions are considered. For solid wall boundary conditions the impermeable no-slip condition is imposed and for lines of symmetry a free-slip, impermeable condition is imposed. Resolving gradients near the walls would require highly refined meshes to resolve flow details in the wall region which is not practical in terms of computer time for application. Therefore, the following turbulent wall conditions are enforced in the first cell away from the wall. It has been shown that these conditions are reasonable for applications characterized predominantly as free-shear flows ${ }^{10}$, such as jet induced mixing. For the $k-\varepsilon$ model, the turbulent kinetic energy, $k$, is set to zero and the dissipation rate, $\varepsilon$, is determined using the Law of the Wall:

$$
\varepsilon=\frac{C_{\mu}^{3 / 4} k^{3 / 2}}{\kappa y} \quad \text { as } \quad y \rightarrow 0
$$

The inlet conditions due to the jet for $k$ - $\varepsilon$ are specified as the turbulent values for fully developed pipe flow presented by Pun and Spalding ${ }^{11}$ :

$$
\begin{aligned}
& k_{\text {inlet }}=0.005\left(\mathrm{U}_{\mathrm{jet}}\right)^{2} \\
& \varepsilon_{\text {inlet }}=C_{\mu} \frac{k^{3 / 2}}{0.03\left(R_{\text {jet }}\right)}
\end{aligned}
$$

The jet is modeled by specifying a Dirichlet velocity condition. 


\section{B. Simulation Validation}

The focus of the present research is to accurately simulate turbulent jets in a low-gravity environment. Due to the limited availability of experimental data for reduced gravity flows, the test matrix is a subset of the drop tower tests performed by Aydelott ${ }^{1}$. This subset includes only fully turbulent jet flows characterized by a jet-Reynolds number greater than $1000^{12}$. The geometry of the tank used in the simulations corresponds to Aydelott's tank 'A', a Plexiglas cylindrical ( $5 \mathrm{~cm}$ radius $\mathrm{x} 20 \mathrm{~cm}$ height) tank with hemispherical end caps. The jet tube has a $0.2 \mathrm{~cm}$ radius and is $1 \mathrm{~cm}$ in height. The fluid properties used in the experiments and simulations correspond to ethanol at $20^{\circ} \mathrm{C}$. A static contact angle of $20^{\circ}$ is specified for ethanol in contact with Plexiglas.

The size of the computational mesh used to model the Aydelott small-scale tank was 44 radial cells by 102 axial cells, or 4,488 total computational cells. This mesh shown in Fig. 2 was demonstrated to give results of comparable accuracy to finer meshes, in particular one 56 by 187 cells, or 10,472 total cells. Any finer mesh would only increase simulation run times without gaining simulation accuracy. Thus, this mesh was employed for all further simulations of the Aydelott small-scale tank. Each rectangle in this mesh represents an annular ring consistent with the cylindrical geometry of the tank. The tank centerline is coincident with the left boundary of the mesh and the right boundary represents the tank wall. The computational simulation employed a no-slip boundary condition at the right

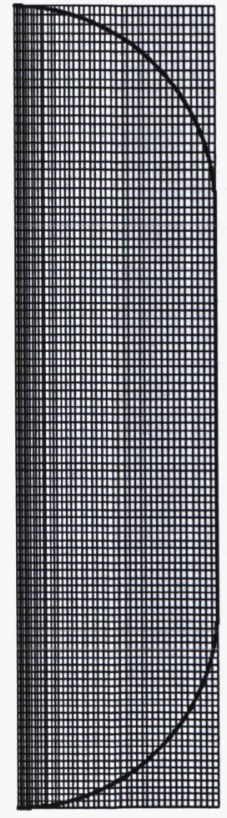

Mesh

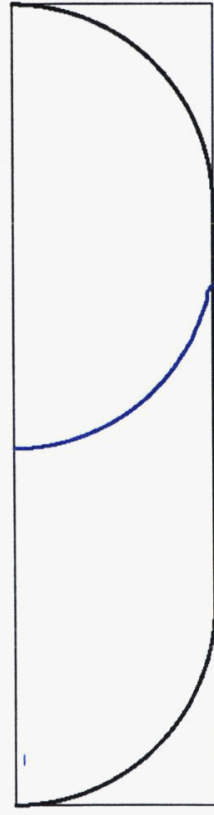

$\mathrm{t}=0 \mathrm{sec}$

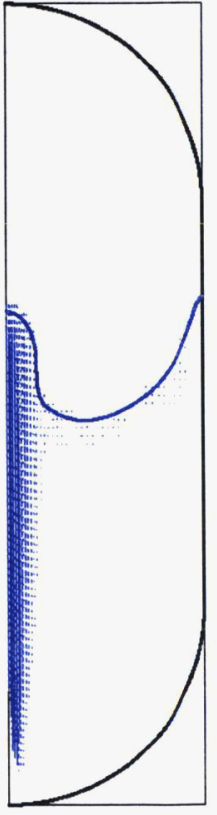

$\mathrm{t}=0.8 \mathrm{sec}$.

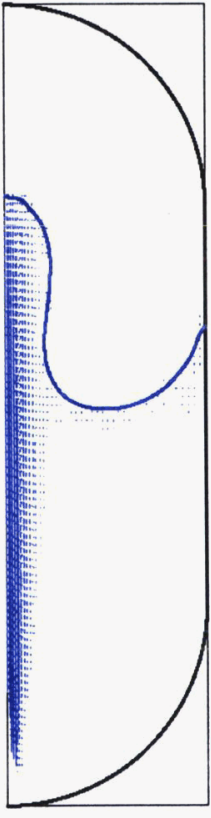

$\mathrm{t}=1.6 \mathrm{sec}$.

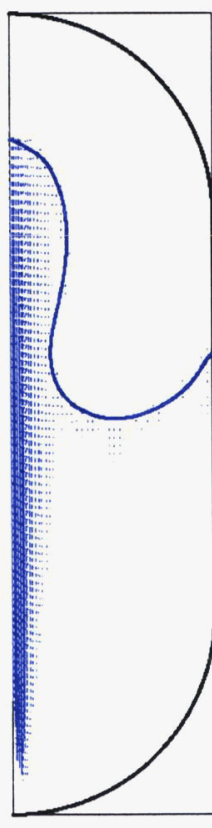

$\mathrm{t}=2.4 \mathrm{sec}$.

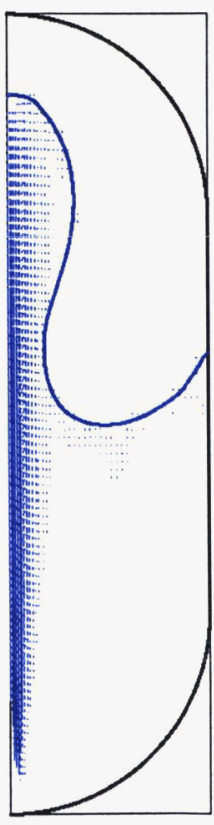

$\mathrm{t}=3.1 \mathrm{sec}$.

Fig. 2 Sample flow field predictions for Pattern II turbulent jet in reduced gravity (Experiment Case 24) 
mesh boundary (tank barrel section) and along the hemispherical end caps. A free-slip condition is imposed at the left mesh boundary. Turbulent boundary conditions were enforced at the jet inlet, Eq. 5 \& 6, and along the solid walls, Eq. 4. To model the experiment conditions, the simulation was initialized with a low gravity equilibrium meniscus. The jet was then activated for 3.1 seconds to match the experiment time. Fig. 2 shows a sample sequence of flow field predictions for a Pattern II turbulent jet in reduced gravity. The blue contour line represents the free surface. The blue regions in the flow fields are composed of velocity vectors.

Table 1 shows the measured and simulated geyser heights for the subset of experiment cases, also displayed in dimensionless form, as a function of the Weber number and Bond number as defined by Aydelott where

$$
\begin{gathered}
B o=\frac{a \rho R_{j}^{2}}{\sigma}=\frac{\text { acceleration force }}{\text { surface tension force }} \\
W e=\frac{\rho V_{o}^{2} R_{o}^{2}}{\sigma D_{j}}=\frac{\text { inertia force }}{\text { surface tension force }} \\
G=\frac{h_{g}}{R_{t}}=\frac{\text { geyser height }}{\text { tank radius }}
\end{gathered}
$$

where $o$ indicates the jet at the pipe outlet, $j$ indicates the jet at the liquid-vapor interface, and $t$ indicates the tank. Also, note that $h_{g}$ is measured from the initial height of the free surface above the jet outlet. To determine the jet radius at the liquid-vapor interface, Aydelott used the following approximate expressions for a turbulent jet derived

\begin{tabular}{|c|c|c|c|c|c|c|c|c|c|c|c|}
\hline $\begin{array}{l}\text { Test } \\
\text { Case }\end{array}$ & $\begin{array}{c}\text { Acceleration } \\
\left(\mathrm{g}^{\prime} \mathrm{s}\right)\end{array}$ & $\begin{array}{l}\text { Liquid } \\
\text { Filling } \\
\text { (\% tank } \\
\text { vol.) }\end{array}$ & $\begin{array}{c}\text { Jet Flow } \\
\text { Rate, } Q \\
(\mathrm{~cm} 3 / \mathrm{s})\end{array}$ & $\begin{array}{c}\text { Jet } \\
\text { Reynolds } \\
\text { Number, } \\
\text { Re }\end{array}$ & $\begin{array}{c}\text { Jet Weber } \\
\text { Number, } \\
\text { We }\end{array}$ & $\begin{array}{c}\text { Jet Bond } \\
\text { Number, } \\
\text { Bo }\end{array}$ & $\begin{array}{c}\text { Simulated } \\
\text { Geyser Height, } \\
\text { hg-comp }\end{array}$ & $\begin{array}{c}\text { Experimental } \\
\text { Geyser Height, } \\
\text { hg }\end{array}$ & $\begin{array}{l}\text { Simulated } \\
\text { Non- } \\
\text { Dimensional } \\
\text { Geyser Height, } \\
\text { Gcomp }\end{array}$ & $\begin{array}{l}\text { Experimental } \\
\text { Non- } \\
\text { Dimensional } \\
\text { Geyser Height, } \\
\text { G }\end{array}$ & $\begin{array}{c}\% \\
\text { Difference }\end{array}$ \\
\hline 6 & 0.005 & 30 & 6.0 & 1290 & 1.51 & 0.22 & 7.40 & 10.20 & 1.48 & 2.04 & 27.4 \\
\hline 7 & 0.0099 & 32 & 6.3 & 1320 & 1.55 & 0.45 & 5.93 & 6.00 & 1.19 & 1.20 & 1.1 \\
\hline 8 & 0.014 & 31 & 6.1 & 1290 & 1.48 & 0.63 & 4.88 & 4.30 & 0.98 & 0.86 & -13.5 \\
\hline 11 & 0.014 & 31 & 8.5 & 1800 & 2.81 & 0.66 & 8.04 & 12.00 & 1.61 & 2.40 & 33.0 \\
\hline 24 & 0 & 52 & 6.3 & 1320 & 1.16 & 0 & 8.66 & 7.25 & 1.73 & 1.45 & -19.4 \\
\hline 25 & 0.0045 & 52 & 6.2 & 1320 & 0.97 & 0.52 & 5.20 & 4.70 & 1.04 & 0.94 & -10.7 \\
\hline 26 & 0.009 & 54 & 6.8 & 1430 & 1.18 & 0.96 & 5.18 & 4.00 & 1.04 & 0.80 & -29.5 \\
\hline 27 & 0.014 & 52 & 6.2 & 1320 & 0.99 & 1.55 & 3.43 & 2.50 & 0.69 & 0.50 & -37.3 \\
\hline 31 & 0.0092 & 53 & 8.7 & 1820 & 1.88 & 1.02 & 7.79 & 7.50 & 1.56 & 1.50 & -3.8 \\
\hline 32 & 0.014 & 51 & 8.7 & 1820 & 1.86 & 1.59 & 6.48 & 6.50 & 1.30 & 1.30 & 0.3 \\
\hline 58 & 0.0022 & 74 & 6.3 & 1320 & 0.74 & 0.43 & 3.60 & 2.50 & 0.72 & 0.50 & -44.1 \\
\hline 62 & 0.014 & 73 & 8.6 & 1820 & 1.38 & 2.88 & 4.78 & 3.80 & 0.96 & 0.76 & -25.7 \\
\hline
\end{tabular}
by Symons and Staskus ${ }^{13}$, which are dependent upon the liquid height above the jet outlet $h_{b}$.

Table 1. Comparison of experimental and computed geyser heights for subset of test cases 


$$
\begin{array}{lll}
R_{j}=R_{o}+0.12 h_{b} & \rightarrow & h_{b} \leq 12.4 R_{o} \\
R_{j}=0.11 R_{o}+0.19 h_{b} & \rightarrow & h_{b}>12.4 R_{o}
\end{array}
$$

These equations are for completely turbulent jets, whereas there are other similar relations for laminar jets and partially turbulent jets.

The correct flow pattern is predicted by the simulation in all cases but one. The geyser height is overpredicted for the 14 test cases by an average of $19.4 \%$, where 10 were overpredicted and 4 were underprected. The challenge, then, is to determine a reasonable threshold for determining whether the simulation yields acceptable predictions as compared to the experiment data. Aydelott performed each case in his experiment test matrix only once and he did not quantify uncertainty. The parallax issues associated with acquiring video data through a cylindrical Plexiglas container suggest that there is a fair amount of uncertainty in the measurements. This uncertainty applies not only to measured and predicted geyser heights, but also to the specification of the initial conditions, which are used in the simulation and based on reported experiment conditions. Yet, he does report dimensionless geyser heights using three significant digits, the apparent resolution of his measurement instrument. Although this level of agreement should be useful for predicting trends, and has been useful for predicting flow patterns, it prompted an investigation into the sensitivity of the computational simulation to user specified parameters; surface tension coefficient, contact angle, and inlet turbulence quantities.

\section{Model Sensitivity}

Aydelott's experiments were conducted in Plexiglas tanks that were carefully cleaned prior to filling with ethanol; even a small amount of contaminant can significantly alter the surface tension coefficient, $\sigma$. The literature does not indicate that any of the physical properties of the fluid were measured and it appears that all calculations related to the experiments have been performed using standard published values for these properties. To study the sensitivity of the simulation to the value of $\sigma$, simulations of a case with a measured geyser height of $6.5 \mathrm{~cm}$ and a computationally predicted height of $3.66 \mathrm{~cm}$ were rerun with $\sigma$ specified to be $90 \%$ and $80 \%$ of the published value for ethanol. Reducing sigma by $20 \%$ resulted in an $8 \%(0.31 \mathrm{~cm})$ increase in computationally predicted geyser height. Although this is a small improvement, it does not fully account for the difference between predicted and measured heights. Further, although the lower value improves the predictions, and the lowered value might be appropriate if there was a slight contamination of the ethanol, there is no evidence in the literature that there was 
contamination and there is no rationale for selecting a specific value for sigma other than the standard published value.

The second parameter to be examined in the sensitivity study is the contact angle between the liquid and the tank wall, $\alpha$. Actual propellant/aluminum/vapor combinations and the experiment ethanol/Plexiglas/air combinations exhibit very small contact angles. To avoid divide-by-zero difficulties in the simulations, the contact angle in the standard simulations has been specified to be 2 degrees. A sequence of simulations was run with increasing values of $\alpha$ for the same test case that was used for the $\sigma$-sensitivity study. Increasing $\alpha$ all the way to 30 degrees resulted in an increase in predicted geyser height of only $0.3 \%$. It is therefore concluded that geyser height is relatively insensitive to contact angle.

The final group of parameters examined as part of the sensitivity study are the turbulence kinetic energy, $\kappa$, and the turbulence energy dissipation rate, $\varepsilon$, at the exit of the jet-pipe. Standard simulations were run using values computed using published correlations for fully developed flow in a pipe. To examine the sensitivity of geyser height to this fairly crude assumption, three additional simulations were performed. The first used values for these parameters that were simply half of the values computed from the correlations. The second and third simulations used values of $\kappa$ selected to produce a turbulent viscosity at the pipe outlet equal to $10 \%$ and $1 \%$ (respectively) of the fully developed value. The increase in geyser height was less than $8 \%$ for any of these cases. Although this is an improvement in the predictive accuracy of the computational simulation, there is no clear rationale for using these reduced values. Again, as concluded for the $\sigma$-sensitivity study, it is comforting to show that geyser height is not particularly sensitive to the specified value of $\kappa$ or $\varepsilon$ and all standard simulations for this study were performed using values computed using the standard correlations for full developed pipe flow.

What conclusions can be reached about the fidelity of the computational model? In the past, the computational model has been asked to simply predict which of the four flow patterns will be produced by a given jet/tank/filling combination and it still does so with good reliability. For the present research, the more challenging task of accurately predicting geyser height for flow pattern II configurations was selected as the fidelity criterion. The computational simulation predicted geyser heights with a maximum overprediction of $33 \%$, a maximum underprediction of $44 \%$, and a mean error of less than $20 \%$. Although the source of this error has not been identified, it seems to be consistent. A sensitivity study to examine the influence of $\sigma, \alpha, \kappa_{\text {inlers }}$ and $\varepsilon_{\text {inlet }}$ on simulated geyser height was conducted because it was believed that these parameters were the most likely to have 
different values between the simulation and the experiment. Although the simulations predictive accuracy could be improved by changing $\sigma, \kappa_{\text {inlet }}$, and $\varepsilon_{\text {inlet }}$ in a reasonable manner, a sound justification for making these changes could not be established. It is reassuring to note that although the predictions improved, the predicted geyser height is not particularly sensitive to these parameters that may not be known á priori with great precision in a spacecraft design environment. Based on all of this information, it was concluded that ECLIPSE is still a very useful tool for predicting jet-induced flow patterns in a propellant tank, that it consistently underpredicts geyser height, and that it should be useful for exploring the relationships between the various parameters that influence geyser production in a low-gravity environment.

\section{Dimensionless Modeling and Prediction}

The objective of dimensional analysis of the geyser phenomena is to establish a correlation to predict geyser formation and geyser height without the expense of continued experimentation. The correlation developed should be general enough to allow its implementation for any combination of tank size, fill level, working fluid, or acceleration level. The development of such a correlation has already been attempted; but like most models and correlations there is room for improvement.

\section{A. Established Correlations}

Through drop tower testing completed in the NASA 5 second zero gravity facility, Aydelott concluded from his data that a linear relationship existed between the non-dimensional geyser height and the Weber number $(\mathrm{We})$. He also assumed that the non-dimensional geyser height should be inversely proportional to the Bond number (Bo). This led to a correlation of the following form:

$$
G=f\left(\frac{X+Y W e}{1+Z B o}\right)
$$

where $X, Y$, and $Z$ are experimentally determined constants and $G$ is the dimensionless geyser height. Through a least-squares curve fitting of experimental data, Aydelott formed the following correlation for completely turbulent jets:

$$
F=\frac{-0.5+1.6 \mathrm{We}}{1+0.6 \mathrm{Bo}}
$$




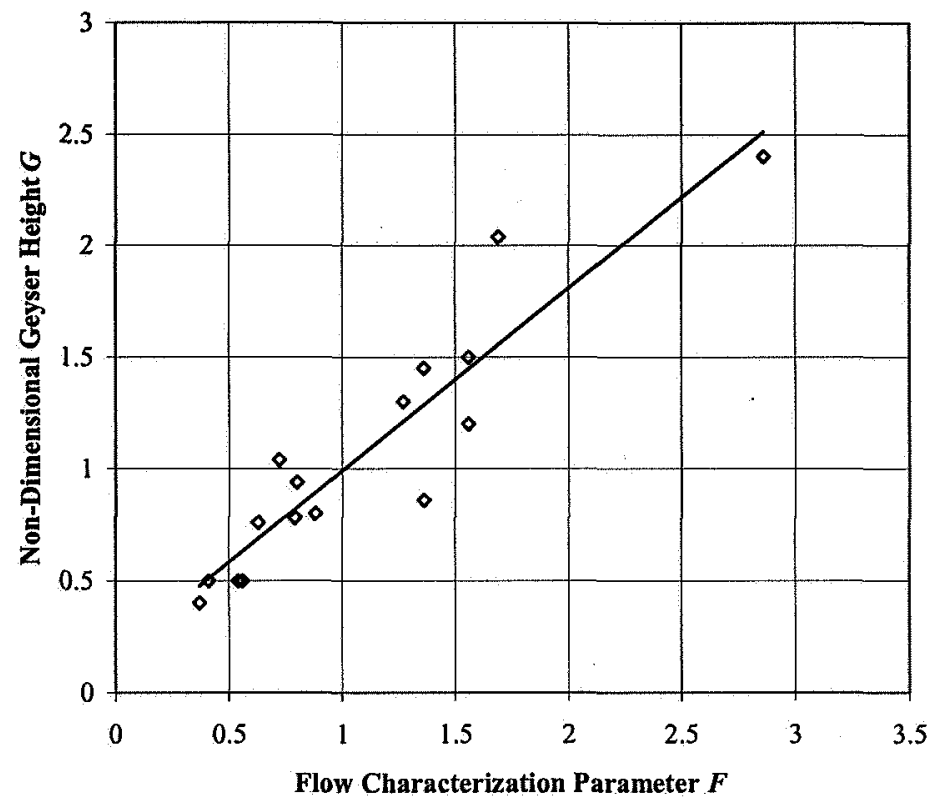

Fig. 3 A graphical comparison between the experiment non-dimensional geyser height and the flow characterization parameter. The solid line is the linear fit of the data points in Eq. 13.

where $F$ is the flow characterization parameter and is expected to equal the dimensionless geyser height $G$. This correlation results in a root mean square (RMS) error of 0.25 between the experimental $G$ and $F$ data provided from the drop tower testing. Fig. 3 is a plot of Aydelott's $G$ vs. $F$ data. Thornton and Hochstein ${ }^{14-16}$ made several attempts at improving Aydelott's correlation culminating in a reformulation of the non-dimensional geyser height. The argument for the modified $G$ was that Aydelott's Bond and Weber numbers indicate the geyser phenomenon was a local effect. That is, the only forces important to the geyser formation were in the narrow vicinity near the jet or geyser. Therefore, the jet radius should be the important dimension, not the tank radius. If this "local" model is accepted then $G$ should be formed as follows:

$$
G_{T H}=\frac{h_{g}}{D_{j}}
$$

This idea was unsuccessful. In fact, the resulting correlation resulted in a larger RMS error for experimental and computational data. 


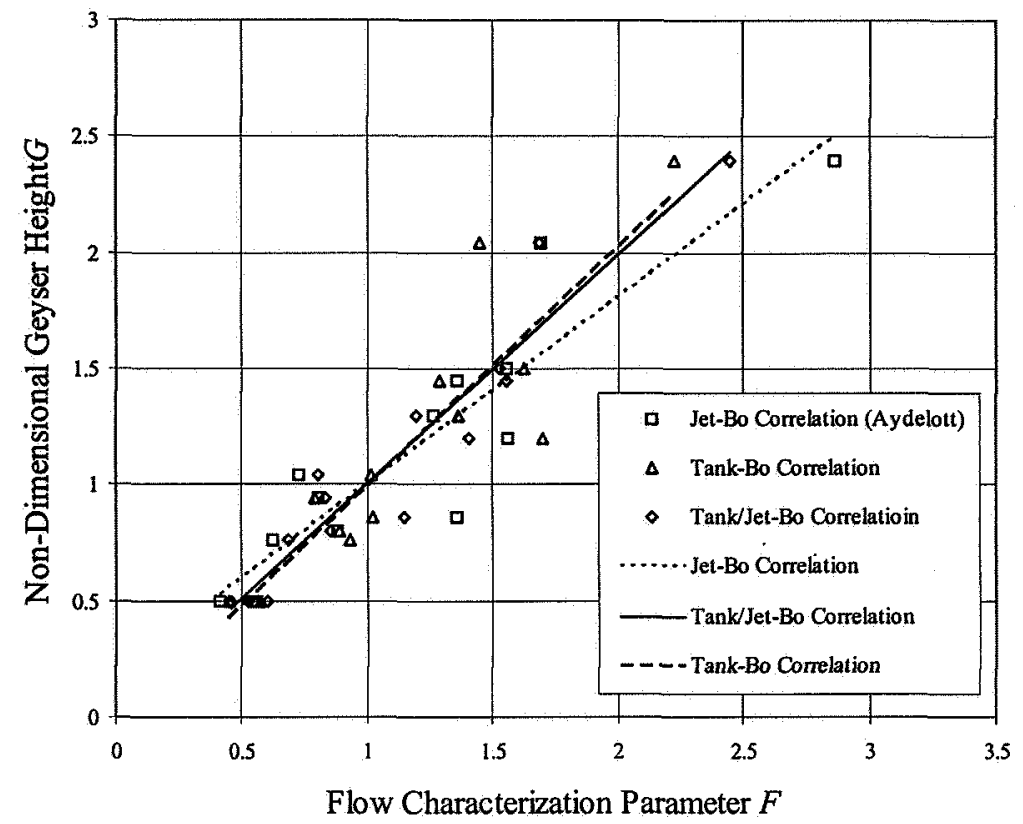

Fig. 4 Correlation using Bond number reformulation.

Thornton and Hochstein also formulated a correlation using a combination jet/tank-Bond number. Here the argument was that depending on the tank to jet diameter ratio, surface tension forces could play varying local and global effects. That is, before the onset of the jet, global effects are dominant as is visible by the large meniscus in the tank. However, once the jet impinges on the free surface, local effects near the geyser itself become quite important. The resulting Bond number and experimental data correlation using a least squares fit to Aydelott's data are as follows.

$$
\begin{gathered}
B o_{i j}=\frac{a \rho\left(D_{i} D_{j}\right)}{\sigma} \\
F_{B o_{y}}=\frac{-0.60+1.86 \mathrm{We}}{1+0.079 B o_{i j}}
\end{gathered}
$$

The result was a reduction of RMS error in geyser height prediction from 0.25 to 0.16 , or nearly a $35 \%$ reduction. Similar results were produced for computational data for the test tank as well as an enlarged tank. Fig. 4 is a graphical representation of these results. The symbols in the figure represent predictions of the flow characterization parameter, $F$, using the improved correlation based on the reformulated $B o$ and $W e$. Straight lines in the figure are linear fits to the data sets generated by each correlation. Data along a 45 degree line would indicate an idea correlation. 


\section{B. Improved Parameter Formulation}

As shown by Thornton and Hochstein ${ }^{9}$, surface tension effects of both local and global scale are likely important in the formation of geysers in propellant tanks. Therefore, the mathematical model used to describe these local and global surface tension forces should include tank and jet dimensions. The previous use of a jet/tank bond number gives equal weight to both local and global effects. However, this may not be the case in the actual physics of the problem. Thus, a logical step to take would be to split up the two parameters and consider separate weighting coefficients.

Here, in an effort to further reduce the RMS error of the geyser correlation, the use of both a jet-Bond number and a tank-Bond number has been made as shown in Eq. 17.

$$
G=f\left(\frac{a+b W e}{1+c B o_{t}+d B o_{j}}\right)
$$

Also, the characteristic lengths have been taken to be the jet and tank diameters rather than the combination of diameters and radii, which Aydelott used. The following are the forms of the dimensionless parameters used in the new correlation.

$$
\begin{gathered}
G=\frac{h_{g}}{D_{t}} \\
B o_{j}=\frac{a \rho D_{j}^{2}}{\sigma} \\
B o_{t}=\frac{a \rho D_{t}^{2}}{\sigma} \\
W e=\frac{\rho V_{o}^{2} D_{o}^{2}}{\sigma D_{j}}
\end{gathered}
$$

Several combinations of the two Bond numbers were investigated. These included both $2^{\text {nd }}$ and $3^{\text {rd }}$ order terms of the Bond numbers. Although the higher order forms improved the prediction accuracy of the correlation, they were dismissed. The small number of data points used to form these correlation raised doubt if the higher order versions were actual improvements. That is, the higher order coefficients may be custom tailoring the correlation to bend in inappropriate ways just to fit the limited set of data points. Thus the version shown in Eq. 17 was used exclusively in this study. 


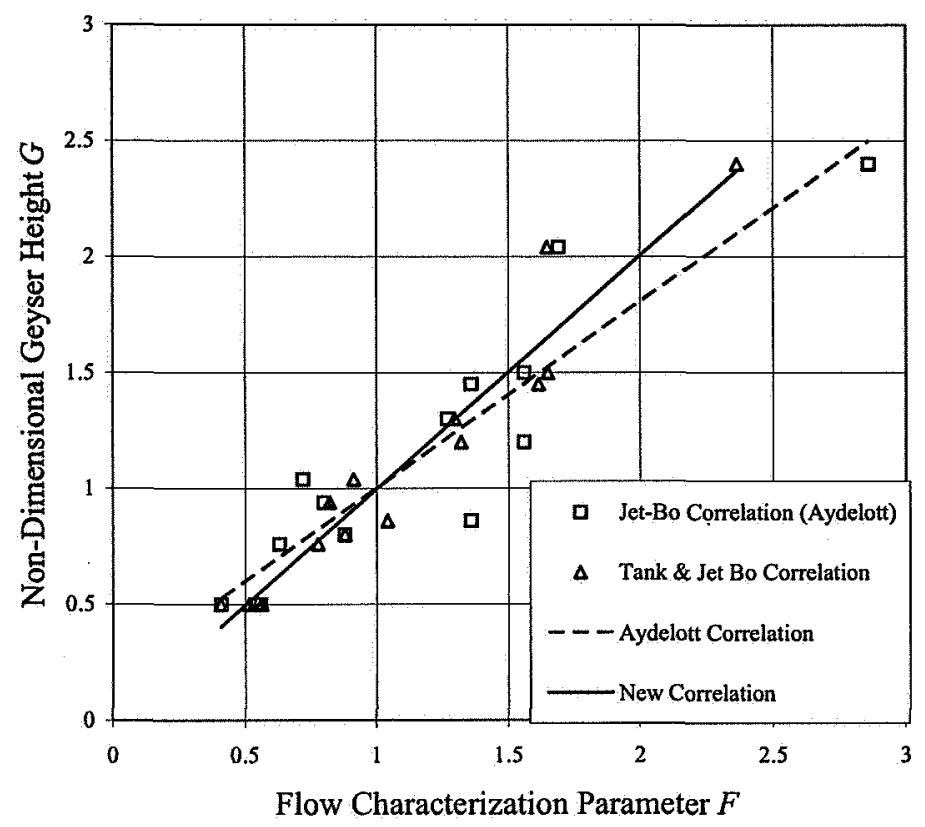

Fig. 5 Graphical plot of experiment data and reformed geyser height correlation.

The drop tower test data presented in Table 1 were used to reformulate the correlation. The coefficients of the correlation were determined using regression. The regression minimized the sum of the squared errors between the measured geyser height and the correlation predicted geyser height and produced the following correlation.

$$
F_{T H}=\frac{-0.9+0.65 W e}{1+0.022 B o_{t}+0.053 B o_{j}}
$$

This correlation yields a RMS error in geyser height prediction of 0.15 compared to 0.25 for Aydelott's original correlation, roughly a $40 \%$ reduction of error. Figure 5 compares the results of the new correlation with the experiment data. It should be noted that data on a 45 degree line would indicate an ideal correlation, that is $F=G$.

\section{Dimensionless Geyser Height Predictions}

Due to the limited amount of experiment data, 12 additional computational cases were added to the original drop tower test data. The cases were chosen to more evenly fill in the jet-Weber and jet-Bond number parameter space. These cases were run using ECLIPSE and were combined with the simulation predictions of the original drop tower 
Table 2. Simulation and correlation predictions for supplemental cases using experiment tank

\begin{tabular}{|c|c|c|c|c|c|c|c|}
\hline $\begin{array}{c}\text { Supplemental } \\
\text { Cases }\end{array}$ & $\begin{array}{c}\text { Jet-Weber } \\
\text { Number, } \\
\text { We }\end{array}$ & $\begin{array}{c}\text { Jet-Bond } \\
\text { Number, } \\
\mathrm{Bo}_{\mathrm{j}}\end{array}$ & $\begin{array}{l}\text { Tank-Bond } \\
\text { Number, } \mathrm{Bo}_{\mathrm{t}}\end{array}$ & $\begin{array}{c}\text { Jet- } \\
\text { Reynolds } \\
\text { Number }\end{array}$ & $\begin{array}{l}\text { Computational } \\
\text { Non- } \\
\text { Dimensional } \\
\text { Geyser Height, } \\
G\end{array}$ & $\begin{array}{c}\text { Aydelott } \\
\text { Correlation, } \\
F_{A Y D}\end{array}$ & $\begin{array}{c}\text { New, } \\
\text { Correlation, } \\
F_{r H}\end{array}$ \\
\hline 1 & 2.00 & 0.00 & 0.00 & 951 & 0.48 & 0.30 & 0.39 \\
\hline 2 & 2.00 & 4.00 & 42.06 & 951 & 0.33 & 0.19 & 0.18 \\
\hline 3 & 2.00 & 8.00 & 84.11 & 951 & 0.28 & 0.14 & 0.12 \\
\hline 4 & 2.00 & 12.00 & 126.17 & 951 & 0.21 & 0.11 & 0.09 \\
\hline 5 & 6.00 & 0.00 & 0.00 & 1647 & 1.32 & 1.19 & 1.39 \\
\hline 6 & 6.00 & 4.00 & 42.06 & 1647 & 0.98 & 0.86 & 0.91 \\
\hline 7 & 6.00 & 8.00 & 84.11 & 1647 & 0.78 & 0.68 & 0.67 \\
\hline 8 & 6.00 & 12.00 & 126.17 & 1647 & 1.85 & 2.19 & $2.60^{*}$ \\
\hline 9 & 10.00 & 0.00 & 0.00 & 2127 & 1.33 & 1.59 & 1.69 \\
\hline 10 & 10.00 & 4.00 & 42.06 & 2127 & 1.13 & 1.25 & 1.26 \\
\hline 11 & 10.00 & 8.00 & 84.11 & 2127 & III or IV & 1.90 & $2.97^{*}$ \\
\hline 12 & 10.00 & 12.00 & 126.17 & 2127 & III or IV & $3.50^{*}$ & $5.55^{*}$ \\
\hline
\end{tabular}

cases. The accuracy of both Aydelott's correlation and the new correlation, Eq. 13 and Eq. 22 respectively, are compared using the RMS error method for all computational data.

The simulated non-dimensional geyser heights for the twelve additional cases are presented in Table 2 . The original and reformulated correlation predictions are also presented in Table 2. An entry of "III or IV" indicates that the geyser simulation contacted the aft end of the tank resulting in flow pattern III or possibly IV. The table also presents the predicted geyser height from both correlations, with an "*" indicating that the predicted height would result in a flow pattern III or IV. Fig. 6 is a plot of the computed geyser heights $G$ verses the correlation predictions $F$ for the original experiment cases and supplemental cases. The new correlation has less data spread from the linear trend than the Aydelott correlation. Again, note that a 45 degree line in this plot would represent an ideal correlation.

The RMS errors for the new correlation and the Aydelott correlation are 0.26 and 0.34 respectively. For the computational results, the new correlation, Eq. 22 , yields nearly a $24 \%$ reduction in error as compared to the original correlation. In addition, the new correlation predicted the two flow pattern III or IV cases while the Aydelott correlation predicted only one. It is also noted that both correlations had one false flow pattern III or IV prediction. 


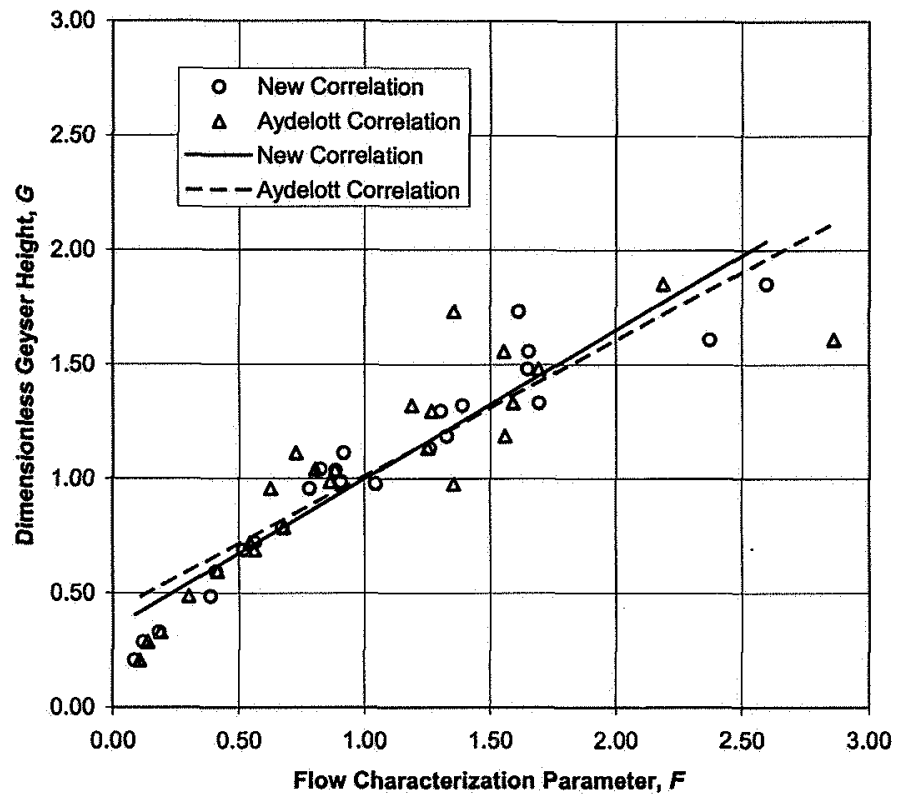

Fig. 6 Correlation precitions using simulation results for 14 experiment cases and 12 sunnlemental cases.

\section{Summary and Conclusions}

Design of future spacecraft propellant management systems employing geyser flows as a component to assist in the suppression of tank self-pressurization will require prediction of geyser formation in the propellant pool. Although an experiment-based correlation exists for predicting jet-induced flow patterns in a sub-scale tank, the data on which this correlation has been developed is very limited due to the high cost of experimentation in a reducedgravity environment. A computational simulation provides a relatively inexpensive alternative to experimentation for gaining insight into the geyser formation process and for developing improved correlations for design.

The fidelity of a computational simulation of jet-induced geyser flows in reduced gravity has been examined. The result of the present dimensional analysis and correlation formulation is an improved geyser prediction tool. The correlation presented in Eq. 22, represents a significant improvement in predictive accuracy over its predecessors. Until further experimental data is collected on geyser formation, this is the most accurate method of predicting geyser heights available. Also, for computational simulation of geysers, ECLIPSE has further demonstrated its capability in providing acceptable results.

It should be noted that the correlation developed here as well as Aydelott's correlation, have been developed using data from experiments in only one small-scale tank. Also, this data was all collected using the same tank 
diameter to jet diameter ratio and test fluid. The applicability of the given correlations to large-scale tanks, different tank to jet diameter ratios, and other fluids is unknown. Thus, a study of these effects is in order. Future work should focus on increasing the quantity of reliable data on geyser formation in a reduced gravity environment. Experimental data will be expensive and time consuming to obtain. If the quality of the computational simulation can be further improved, it may provide a less expensive path to acquiring the necessary information. The new correlations developed during the present study show clearly improved performance for the available data.

\section{References}

${ }^{1}$ Aydelott, J.C., "Axial Jet Mixing of Ethanol In Cylindrical Containers During Weightlessness," NASA TP-1487, 1979.

${ }^{2}$ Aydelott, J.C., "Modeling of Space Vehicle Propellant Mixing," NASA TP-2107, 1983.

${ }^{3}$ Poth, L.J., and Van Hook, J.R., "Control of the Thermodynamic State of Space-Stored Cryogens by Jet Mixing," Journal of Spacecraft, Vol. 9, No. 5, May 1972, pp. 332-336.

${ }^{4}$ Jones, W.P., and Launder, B.E., "The Prediction of Laminarization with a Two-Equation Model of Turbulence," International Journal of Heat and Mass Transfer, Vol. 15, pp. 301-314, 1972

${ }^{5}$ Pope S.B., "An Explanation of the Turbulent Round Jet/Plane Jet Anomaly," AIAA Journal, Vol. 16, No. 3, March 1978, pp. 279-281. Hochstein, J.I., Gerhart, P.M., and Aydelott, J.C., "Computational Modeling of Jet Induced Mixing of Cryogenic Propellants in Low-G," AIAA Paper 1984-1344, 1984.

${ }^{6}$ Kothe, D.B., Mjolsness, R.C., and Torrey, M.D., "Ripple: A Computer Program for Incompressible Flow with Free Surfaces," Los Alamos National Laboratory, LA-12007-MS, 1991.Messerole, J.S., "Mixing Induced Ullage Condensation and Fluid Destratification," AIAA Aerospace Sciences Meeting Paper 87-2018, 1997.

${ }^{7}$ Hochstein, J.I., Gerhart, P.M., and Aydelott, J.C., "Computational Modeling of Jet Induced Mixing of Cryogenic Propellants in Low-G," AIAA Aerospace Sciences Meeting Paper 1984-1344, 1984.

${ }^{8}$ Hochstein, J.I., "Computational Modeling of Jet-Induced Geyser Mixing in Cryogenic Propellant Tanks in Low-G," Doctoral Dissertation, University of Akron, 1984

${ }^{9}$ Hochstein, J.I., Gerhart, P.M., and Aydelott, J.C., "Computational Modeling of Jet Induced Mixing of Cryogenic Propellants in Reduced Gravity," AIAA Aerospace Sciences Meeting Paper 91-0801, 1991.

10 Wilcox, D.C., Turbulence Modeling for CFD, $2^{\text {nd }}$ Edition, La Canada, DCW Industries Inc., 2000.

$"$ Pun, W.M., and Spalding, D.B., "A General Computer Program for 2-Dimensional Elliptic Flows," Imperial College of Science and Technology, 1977.

12 Birkoff, G., and Zarantonello, E.H., Jet, Wakes, and Cavities, Academic Press, 1957. 
${ }^{13}$ Symons, E.P., and Staskus, J.V. "Interface Stability During Liquid Inflow to Partially Full Hemispherical Ended Cylinders During Weightlessness." NASA TM X-2348, 1971.

14 Thornton, R.J., "A Computational and Dimensional Analysis of Microgravity Propellant Tank Geyser Prediction," Magisterial Thesis, The University of Memphis, Memphis, TN, 2000.

15 Thornton, R.J., and Hochstein, J.I., "Microgravity Geyser and Flow Field Prediction," AIAA Aerospace Sciences Meeting Paper 2000-0858, Jan 2000.

16 Thornton, R.J., and Hochstein, J.I., "Microgravity Geyser Analysis and Prediction," AlAA Aerospace Sciences Meeting Paper 2001-1132, Jan 2001. 